\title{
Publisher's Note: Theory of magnon motive force in chiral ferromagnets [Phys. Rev. B 94, 020405(R) (2016)]
}

Utkan Güngördü and Alexey A. Kovalev

(Received 20 July 2016; published 28 July 2016)

DOI: 10.1103/PhysRevB.94.019901

This paper was published online on 13 July 2016 with errors in Refs. [55] and [57].

Reference [55] should read as "While a rigorous analysis of domain wall dynamics in the presence of a strong DMI should take domain wall tilting into account in general, in the particular case of a domain wall driven by a perpendicular field, the $(X, \phi)$ model remains moderately accurate for studying the effects of feedback damping [16,68]."

Reference [57] should read as "This equation is similar to the equation obtained for electronic feedback damping in [16] but for magnons $\Gamma_{X X}$ and $\Gamma_{\phi \phi}$ are determined by $D / J$ rather than $\tilde{\alpha}_{R}$ (a parameter which is taken to be independent from $D$ ), which leads to different conclusions. In [16], the chiral derivative associated with SMF is parametrized by $\tilde{\alpha}_{R}$ as $\mathcal{D}_{i}=\partial_{i}+$ $\left(\tilde{\alpha}_{R} \boldsymbol{e}_{z} \times\right) \boldsymbol{e}_{i} \times$. For magnons, $D / J$ corresponds to $\tilde{\alpha}_{R} . "$

The references have been corrected as of 20 July 2016. The references are correct in the printed version of the journal. 